\title{
Magnetic Solitons in a Bose-Einstein Condensate
}

\author{
Two independent experiments generate self-reinforcing magnetic waves \\ in a condensate containing both spin-up and spin-down atoms.
}

\section{By Christopher Crockett}

$\mathrm{M}$ ost waves lose their shape as they travel, but solitons defy that behavior. These self-reinforcing waves are ubiquitous in natural and artificial environments such as rivers, optical fibers, and even Bose-Einstein condensates (BECs; see Synopsis: Solitons of All Speeds). Now, two teams of researchers have independently created magnetic solitons in a $\mathrm{BEC}$ made from atoms with different spins. The experiments establish a new playground for further exploration of quantum solitons.

The teams were led by Giacomo Lamporesi of the University of Trento in Italy and Chandra Raman of the Georgia Institute of Technology, Atlanta. While the details vary, both teams used similar experimental setups. They prepared an elongated BEC with a 50-50 mix of intermingled spin-up and spin-down sodium atoms. They then fired a laser pulse at the BEC. The laser's illumination pattern and wavelength were chosen to "kick" the spin-up and spin-down atoms in opposite directions: for a

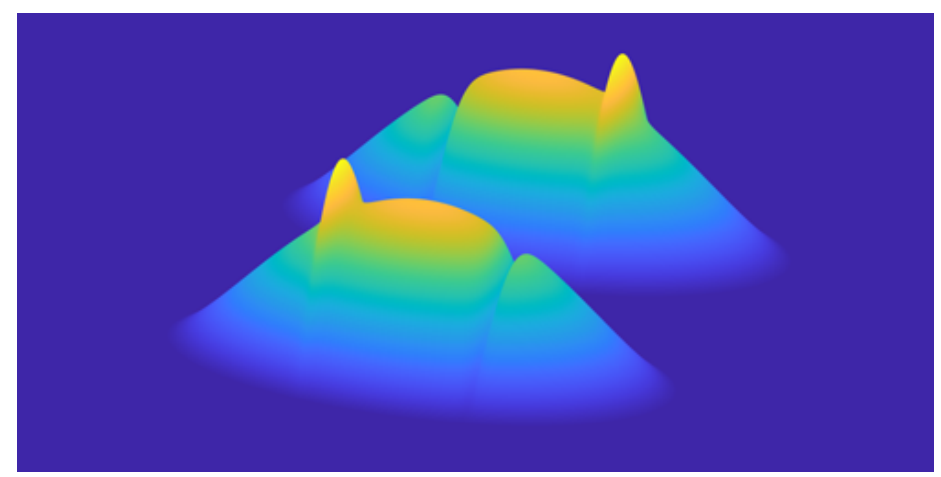

Credit: X. Chai/Georgia Institute of Technology particular choice of circular polarization, the spin-down atoms were drawn toward the light, while the spin-up atoms were repelled. This effect sent a slow-moving, self-reinforcing ripple through the spin-up atoms in one direction and a similar ripple through the spin-down atoms in the other direction.

Both teams also engineered "collisions" between the magnetic solitons by altering the laser pulse to generate pairs of solitons at opposing ends of the BEC. When the solitons met in the middle, they passed right through one another. Raman says that he and his team hope to explore such scenarios in more detail, looking for situations where the solitons get distorted. They also plan to generate trains of magnetic solitons to see how they interact. Lamporesi's group hopes to expand this research to $2 \mathrm{D}$ and $3 \mathrm{D}$ systems, where structures in the $\mathrm{BEC}$, such as magnetic vortices, could be leveraged to mimic some phenomena in high-energy physics.

This research is published in Physical Review Letters.

Christopher Crockett is a freelance writer based in Arlington, Virginia. 